\title{
Calculation of Lung Volume in Newborn Infants by Means of a Computer-assisted Nitrogen Washout Method
}

\author{
BENGT ARNE SJÖQVIST, KENNETH SANDBERG, OLA HJALMARSON, AND TORSTEN OLSSON \\ Research Laboratory of Medical Electronics, Chalmers University of Technology [B.A.S., T.O.] and Department \\ of Pediatrics I, University of Göteborg [K.S., O.H.], Göteborg, Sweden
}

\begin{abstract}
A clinically adapted method for the calculation of the functional residual capacity in newborn infants has been developed. The method is based on a multiple breath nitrogen washout test, during which the ventilatory air flow and the nitrogen concentration signals are sampled by a minicomputer, which also performs the calculations. The ventilatory air flow is measured by a pneumotachometer connected to a facemout volume displacement body plethysmograph, and the nitrogen concentration by a nitrogen analyzer. The functional residual capacity volume is calculated from the sampled signals by adding the expired nitrogen volumes during each expiration, and finally dividing this sum by the initial alveolar nitrogen concentration. Before the calculations, the sampled signals are adjusted regarding nitrogen analyzer delay and plethysmograph characteristics. The method presented is designed to minimize the test equipment influence on the baby's respiration and also to inhibit the necessity of pneumotachometer compensations normally connected with washout methods. Furthermore, the calculated breath-by-breath values of end-expiratory nitrogen concentration, nitrogen volume, inspired and expired tidal volume, are stored on disk for further analysis and resimulation of the test. The method has been tested on a mechanical lung model and on washouts from healthy newborn infants. The model tests indicate that the accuracy and the reproducibility of the method are good, and the results from the infants are in good agreement with previously obtained results. (Pediatr Res 18:1160-1164 1984)
\end{abstract}

\section{Abbreviations}

FRC, functional residual capacity TGV, thoracic gas volume

Following birth, the fetal lung fluid is gradually replaced by air, and an adequate lung function is soon established. Normally, this is an extremely efficient process. Disturbances in this process, e.g. the inability to develop an appropriate lung volume, lead to neonatal respiratory disease. The extent to which an adequate. lung volume has been established can be determined by meas-

Received November 11, 1983; accepted April 24, 1984.

Requests for reprints should be addressed to B. A. Sjöqvist, Research Laboratory of Medical Electronics. Chalmers University of Technology, S-412 96 Göteborg, Sweden.

This work was supported by the Swedish Medical Research Council, Project B80-19X-05703, the Medical Faculty of the University of Göteborg, and the Research Fund of the Children's Hospital, Göteborg. uring FR'C. Despite its central importance, measurements of FRC are seldom used clinically in neonatal medicine, because of the lack of suitable methods.

Therefore, in order to facilitate such measurements, we have developed a clinically adapted computer-assisted plethysmographic nitrogen washout method for the calculation of FRC in newborn infants, which reduces the problems connected with ordinary washout methods $(3,8,15)$. It also enables further analysis of the collected washout data, for instance nitrogen elimination pattern ("distribution of ventilation") analysis. The method has been tested on a group of healthy newborn infants and on a mechanical lung model. TGV, calculated according to DuBois et al. (5), was used as reference in the studied infants. The reproducibility of the measurements was tested in infants and on a mechanical lung model. This lung model was also used to test the accuracy of the FRC calculation method.

\section{METHOD AND THEORY}

The system is illustrated in Figures 1 and 2. It consists of a face-out volume displacement body plethysmograph (Fig. 1), a minicomputer, and equipment for measurement of ventilatory air flow and breathing gas nitrogen concentration (Fig. 2). The plethysmograph is constructed from transparent plexiglas and has previously been described in detail (10). The ventilatory air flow is measured by a pneumotachometer, Fleish No. 1, connected to a small aperture in the plethysmograph wall, and a differential pressure transducer (Elema-Schönander EMT 32). The breathing gas nitrogen concentration is measured by a Hewlett-Packard 47302A nitrogen analyzer having a rise time of $30 \mathrm{~ms}$ and a delay time to the $10 \%$ point of less than $50 \mathrm{~ms}$. The analyzer probe and a Rendell-Baker face mask are connected to a T-tube, through which a constant gas flow is maintained. The face mask is partly filled with grease (Macrogol) in order to diminish the dead space and to make the system air-tight. The dead space of the whole system is approximately $2 \mathrm{ml}$. A polygraph (Mingograph, Siemens-Elema) is used for monitoring the ventilatory flow and nitrogen concentration signals.

An examination is performed with the baby lying in the plethysmograph, and the face mask gently placed over the resting or sleeping baby's nose and mouth. During an expiration, the gas flow in the mask is changed to $100 \%$ oxygen and a nitrogen washout test is performed. When the nitrogen concentration in the expired air stays below $2 \%$, the gas flow is returned to the original breathing air composition and the test is finished. The ventilatory air flow and the nitrogen concentration signals (Fig. 3) are sampled by the computer throughout the test and stored on disk for the calculations which follow.

The sampling frequency is $250 \mathrm{~Hz}$, corresponding to a sample interval of $4 \mathrm{~ms}$, and the signals are filtered by a fourth order 


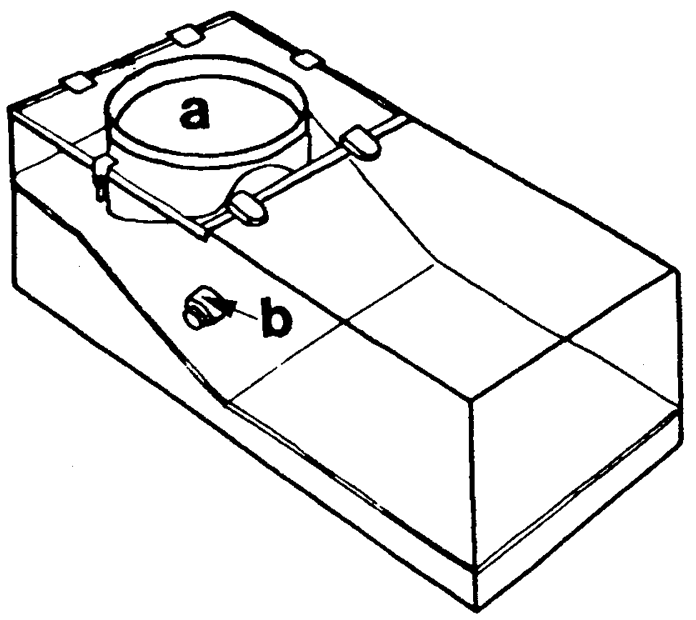

Fig. 1. The plethysmograph, where $a$ indicates the position of the baby's face, and $b$ is the pneumotachometer connection.

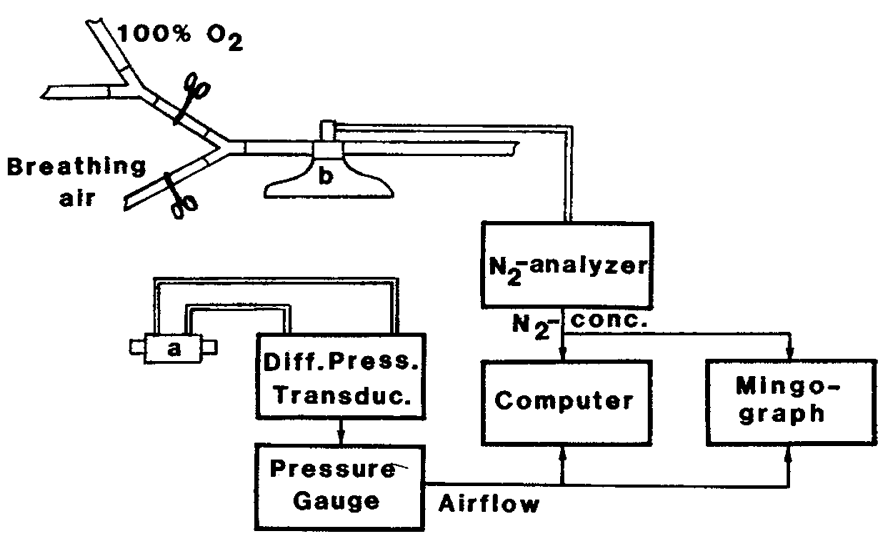

Fig. 2. The test arrangement, where $a$ is the pneumotachometer and $b$ is the face-mask.

\section{Airflow

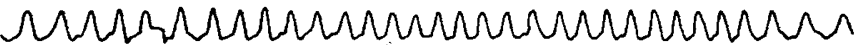

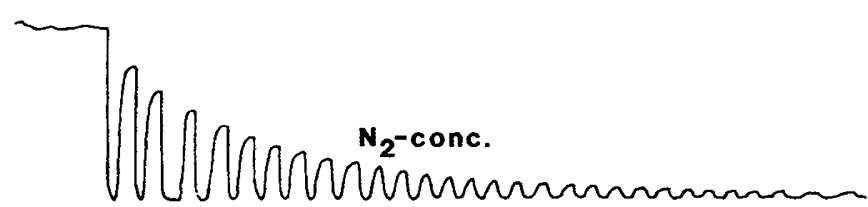

Fig. 3. A representation of the air flow and the nitrogen concentration during a washout test.

Butterworth low-pass filter with a cut-off frequency of $60 \mathrm{~Hz}$ before the sampling. The minicomputer is a PDP 11/40 and the software is written in FORTRAN. During the computer calculations, the instantaneous product of the sampled flow and nitrogen concentration is digitally integrated during each expiration and inspiration in order to obtain the volumes of expired and inspired nitrogen (Fig. 4). The nitrogen volumes expired during the washout are added until the breath after which the end-expiratory nitrogen concentration stays below $2 \%$, and the sum is finally divided by the end-expiratory nitrogen concentration prior to the oxygen breathing. This concentration approximates the initial alveolar concentration. The result is the FRC volume given in body temperature and pressure, saturated with water vapor. Besides the expired nitrogen volumes, the breathby-breath end-tidal nitrogen concentrations, expired and inspired

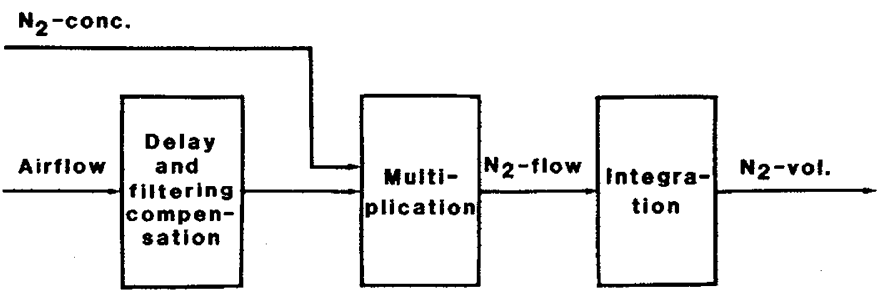

Fig. 4. The nitrogen volume calculation principle.

tidal volumes, are calculated and stored on disk, where they are accessible for resimulation of tests and other programs.

Before multiplication, the two sampled signals are adjusted by the computer with respect to nitrogen analyzer delay and plethysmograph characteristics. The nitrogen analyzer delay is approximately $75 \mathrm{~ms}$ and was determined by introducing a flow of $100 \%$ oxygen into a tube, to which the gas analyzer probe and the pneumotachometer were attached. The delay was calculated from the resulting flow and concentration signals, both by computer analysis of the sampled signals and through visual inspection of an analog plot. The low-pass filtering influence of the plethysmograph, which introduces a delay in the flow signal and thereby reduces the total delay between the signals, is compensated for by using the derivative of the recorded air flow and the time constant of the plethysmograph, according to Ref. 10.

$$
V_{i}^{\prime}=V_{p n}^{\prime}+R C V_{p n}^{\prime \prime}
$$

where $V_{l}^{\prime}$ is the flow initiated by the thorax movement, $V_{p n}^{\prime}$ is the pneumotachometer flow, $R C$ is the time constant of the plethysmograph, and $V_{p n}^{\prime \prime}$ is the time derivative of $V_{p n}^{\prime}$. By using an appropriate $R C$ value in this compensation, the desired 75ms total delay betwen the sampled signals is obtained. The $R C$ value has previously been determined as $30 \mathrm{~ms}(10)$. The filtering compensation of the air flow is made continuously in the computer programs, before the multiplication with the correctly delayed concentration value is performed. The delay value is 76 $\mathrm{ms}$ and is chosen in order to correspond with the sample interval of $4 \mathrm{~ms}$. No correction is made in the calculations for the corresponding time constant of the respiratory system, since it is smaller than, and well separated from, the plethysmograph constant and therefore assumed not to affect the results (10).

A flow and a nitrogen concentration calibration signal are generated on every test occasion. The flow signal is obtained from a calibrated rotameter, and the concentration signal originates from the nitrogen analyzer, sampling room air. The calibration signals are sampled by the computer and used for calibration in the calculation routines. No corrections are made in the calculations for the elimination of tissue-solved nitrogen. The method and the calculations, including the software, are described in detail elsewhere (19).

In order to evaluate the method during predetermined conditions, a mechanical lung model, according to the plan in Figure 5 , has been constructed. This model consists of three Plexiglas disks, $0.5 \mathrm{~mm}$ thick, attached to each other, and a loudspeaker for generation of the ventilatory oscillations. The mixing chamber, i.e. the lung volume, consists of a $160-\mathrm{mm}$ wide hole in the middle disk, which is limited by a membrane attached above a corresponding hole in the bottom disk and a top disk with a gas outlet-inlet. The membrane, made of latex rubber, performs the ventilatory oscillations by using mechanical transmission from the loudspeaker. The total lung model volume is $107 \mathrm{ml}$. The lung model can be ventilated with varying tidal volumes and frequencies by using a signal generator connected to the loudspeaker.

The mechanical model has been used to test the reproducibility and the accuracy of the calculated volumes. In these tests, the complete construction was fitted into the plethysmograph using the same test arrangement as in Figure 2, and thereby simulating the clinical measuring situation (Fig. 6). Ten washout tests were 


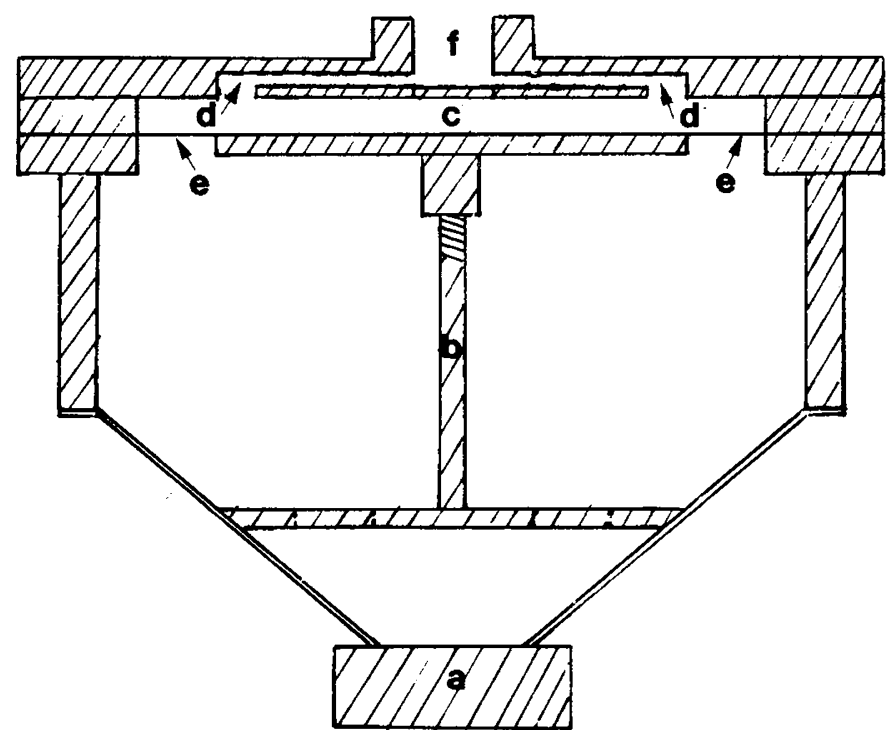

Fig. 5. A cross-section of lung model, where $a$ is the loudspeaker, $b$ is the mechanical transmission, $c$ is the mixing chamber, $d$ are airchannels, $e$ is the latex membrane, and $f$ is the gas inlet-outlet.

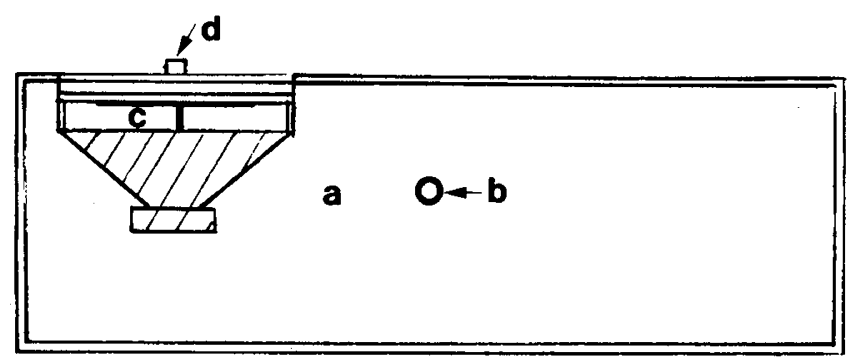

Fig. 6. The lung model fitted into the plethysmograph, where $a$ is the plethysmograph, $b$ is the pneumotachometer connection in the plethysmograph wall, $c$ is the lung model, and $d$ is the gas inlet-outlet of the mixing chamber.

performed. The frequency and the tidal volume were held at approximately constant values, the frequency was 50 breaths/ min, and the mean tidal volume was $18.6(\mathrm{SD}=0.3) \mathrm{ml}$. The theoretical total lung volume at midexpiration was $107.3 \mathrm{ml}$, and the theoretical FRC value was thus $98.0 \mathrm{ml}$, i.e. the total volume minus half the tidal volume. The FRC volumes were calculated by using all the expired nitrogen volumes until the concentration was well below $1 \%$, typically 0.5 to $0.7 \%$, and appropriate delay compensation.

The reproducibility was further tested in a group of nine infants by repeating the test twice with an interval ranging from 15 to $35 \mathrm{~min}$ between the measurements. The accuracy of the method was also evaluated in newborn infants by comparing the calculated FRC volumes with simultaneously measured TGV. The TGV measurement was performed with the baby still resting in the plethysmograph. The cheek and submandibular tissues were supported by a stiff cuff (10). A rigid face mask connected by a rigid tube to a pressure transducer (Elema-Schönander, EMT 34) was placed over the infant's nose and mouth. At the end of an expiration, the port of the mask was closed manually. The mouth pressure and body volume variations were recorded on the polygraph during three to five consecutive breaths. At least three successful determinations with pressure and volume signals in phase during airway closure were made in each infant. The mean TGV was then calculated according to DuBois et al.

After parental consent, 40 healthy newborn infants were examined on 64 occasions, at ages between 1.5 and $144 \mathrm{~h}$. They were delivered vaginally or by cesarian section. Sixteen of these infants had recovered from respiratory disease. The median birth weight was $3.36 \mathrm{~kg}$ (range, $1.85-4.30 \mathrm{~kg}$ ) and the median gestational age was $39 \mathrm{wk}$ (range, 33-42 wk).

\section{RESULTS}

The reproducibility and the accuracy of the method were tested by using the mechanical lung model and resulted in a mean value of the calculated FRC of $96.2 \mathrm{ml}$, a standard deviation of $2.8 \mathrm{ml}$, and a coefficient of variation of $2.9 \%$. This corresponds to a $95 \%$ confidence interval from 94.2 to $98.2 \mathrm{ml}$. The theoretical FRC was $98.0 \mathrm{ml}$.

The reproducibility was also tested in nine newborn infants. The results are presented in Figure 7. The correlation coefficient was $r=0.92(p<0.001)$ and the mean difference between the examinations was $3.6 \mathrm{ml}$, with a $95 \%$ confidence interval from -2.2 to $9.4 \mathrm{ml}$.

The calculated mean FRC in the group of 40 infants, 64 measurements, was $67.2(\mathrm{SD}=18.1) \mathrm{ml}$, and the corresponding TGV was $84.9(\mathrm{SD}=19.6) \mathrm{ml}$. The mean difference between TGV and FRC was thus $17.7(\mathrm{SD}=16.7) \mathrm{ml}$. A paired $t$ test showed that the difference between FRC and TGV was statistically significant $(p<0.001)$. A comparison of the FRC and TGV values can be seen in Figure 8. The correlation coefficient was $r$ $=0.69(p<0.001)$.

\section{DISCUSSION}

Several problems are involved when gas technique methods are designed for lung volume measurements in neonates. The amount of gas to be measured is often very small, and even a minor leak will consequently cause a great error. It is also important to keep the "dead space" of the measuring system as small as possible in order to minimize the influence on the respiration. The general problems of pneumotachometer variation and compensation according to temperature and gas composition (21) also have to be taken into account, as well as the impossibility of getting any cooperation from the baby during the test. In the method presented in this paper, these problems have been carefully considered and the method has a number of advantages, although the FRC calculation principle (Fig. 4), based on the sampled signals, is similar to previously described methods $(3,15,17,18)$.

The use of a plethysmograph for ventilatory air flow measurement implies that the flow through the pneumotachometer con-

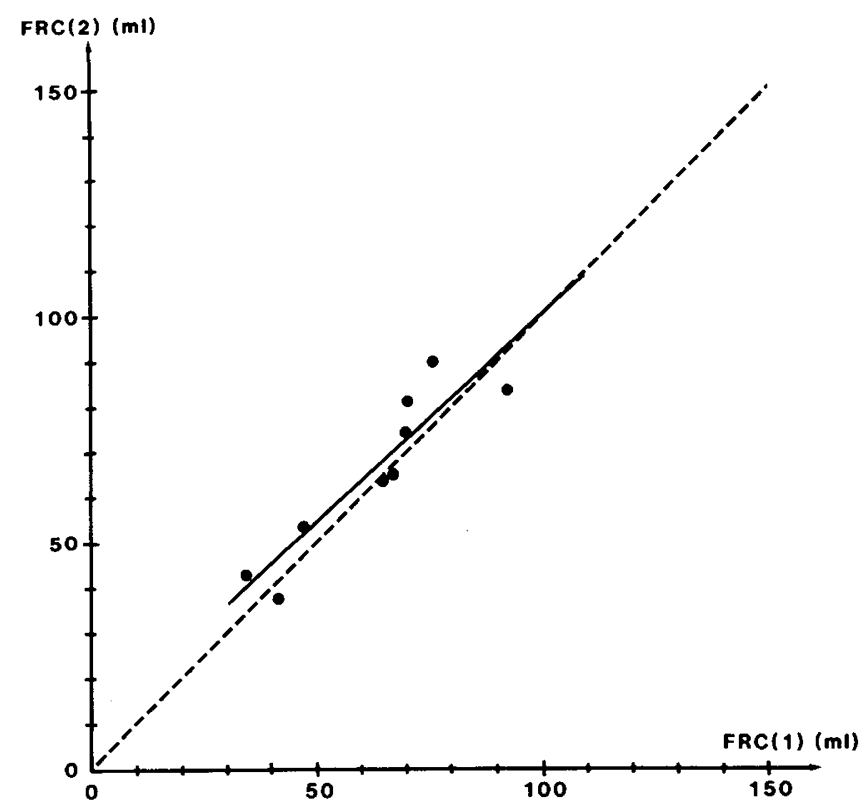

Fig. 7. Relationship between two consecutive calculations of FRC in nine newborn infants $(F R C(2)=9.5+0.9 \times F R C(1)$. 


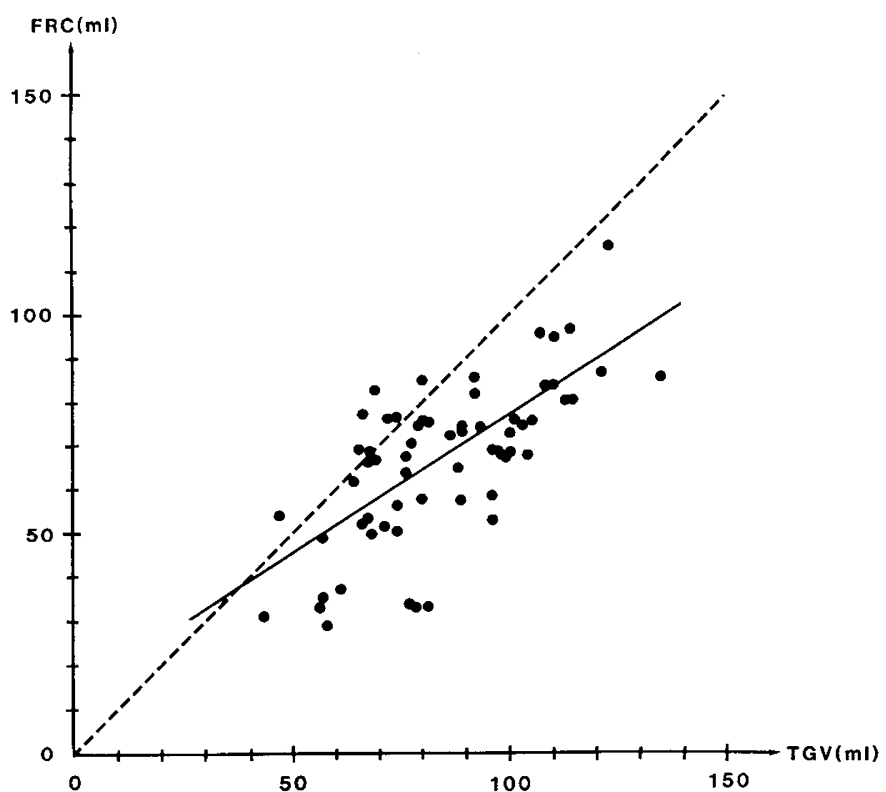

Fig. 8. Relationship between calculated FRC and TGV in 64 simultaneous measurements $(\mathrm{FRC}=13.3+0.6 \times \mathrm{TGV})$.

sists of gas with approximately constant temperature and composition, and therefore no temperature or viscosity compensation to the recorded air flow signal is necessary. These variations may be as large as 10 to $15 \%$ during a test if the pneumotachometer is placed in the breathing air gas stream (21). The use of the plethysmograph also implies that the calculated volumes are automatically given in body temperature and pressure (saturated) and that TGV and basal ventilation can be measured during the same session. Furthermore, the plethysmograph and the facemask modifications have reduced the equipment dead space, and thereby reduced its influence on the ventilatory pattern. By using $100 \%$ oxygen for the test, air contamination due to leaks and inappropriate washout starts are easily detected from the nitrogen concentration signal. When applying the method, however, it is necessary to consider the time constant of the plethysmograph. The time constant is approximately $30 \mathrm{~ms}$ and introduces phase distortion in the flow signal in the actual frequency domain, i.e. around $1 \mathrm{~Hz}$. This distortion is compensated for by using the previously presented Equation 1. By using the initial end-expiratory nitrogen concentration in the FRC calculations, the method becomes independent of oxygen therapy given to the test subject, whereas the calculations are always based on actual breathing air concentration. Finally, the use of a computer, data reduction, and storing of the resulting breath-by-breath endexpiratory nitrogen concentrations, expired nitrogen volumes, expired and inspired tidal volumes, make it possible to analyze further and recapitulate the washout test. It is also worth noticing that if, for a certain project, the advantages of the plethysmograph and the reduced dead space can be neglected, the flow signal for the calculations can as well be measured by a pneumotachometer in the mask system.

No corrections have been made in the calculations for the elimination of tissue nitrogen that takes place during the $100 \%$ oxygen breathing. If studies of tissue nitrogen elimination during pure oxygen breathing in adults and animals $(7,12,16)$ can be applied to newborn infants, the calculated FRC volume is overestimated by less than $1 \mathrm{ml} / \mathrm{kg}$ body weight. The overestimation of FRC is probably further reduced while the calculations are stopped at the $2 \%$ level, where all the initial nitrogen is not eliminated.

The tests on the mechanical lung model indicate that the reproducibility and the accuracy of the method are good. Ten consecutive measurements gave a $95 \%$ confidence interval which included the true FRC volume. As the washout tests were stopped
Table 1. FRC $\left(\mathrm{N}_{2}\right)$ in comparison with previous studies: $F R C$ values are adjusted to $3.4 \mathrm{~kg}$ body weight

\begin{tabular}{lccc}
\hline & Body weight $(\mathrm{kg})$ & Age $(\mathrm{h})$ & FRC $(\mathrm{ml})$ \\
\hline Nelson et al. $(13)$ & $1.3-4.1$ & $16-1704$ & 72.9 \\
Strang et al. $(20)$ & $2.2-3.9$ & $5-144$ & 52.4 \\
Prod'hom et al. $(14)$ & $2.3-4.3$ & 24 & 83.0 \\
Hanson et al. $(8)$ & $1.9-4.3$ & $1.75-128$ & 57.8 \\
Present study & $1.9-4.3$ & $1.5-144$ & 67.2 \\
\hline
\end{tabular}

when the "end tidal nitrogen concentrations" were approximately $0.5 \%$ the remaining nitrogen in the "lung" would further reduce the difference between the estimated and the true mechanical "lung" volume.

The reproducibility test in infants indicates some of the problems involved in this kind of measurement. The results show a good correlation between the two measurements, but there is some individual variation. The two FRC measurements were performed with the baby at quiet sleep, as judged from the breathing pattern, and at times 15-35 min apart, in order to allow equilibration between alveolar gas and breathing air. However, it is known from adults that cyclical variations in FRC exist during normal breathing (11), and it has also been suggested that such variations can occur in infants as a consequence of different sleep stages (9). During the time between the measurements, it is thus possible that a change in lung volume may have occurred, resulting in the observed individual variation.

The TGV, measured according to DuBois et al., was used as reference method. There was a significant correlation between FRC and TGV $(r=0.69, p<0.001)$. However, as in previous studies (13), we found considerable differences between FRC and TGV. This phenomenon has often been attributed to the presence of trapped gas within the infant's lung. Whether this is true or whether this difference can be caused methodologically has recently been questioned (1). The justification for using pure oxygen as diluting gas during FRC measurements has also been questioned (6). However, since the method measures the nitrogen content of the lung at the onset of the test, the calculated FRC represents the correct lung volume, as long as no parts of the lung become completely isolated, i.e. their gas transport becomes completely inhibited, due to the oxygen breathing. In a recent report, it has been proposed that pure oxygen breathing for about 3 min has little or no effect on the FRC (4). A comparison with previously published FRC values determined by means of nitrogen methods are presented in Table 1 . Our results are in the same range, and the discrepancies could be due to methodological differences and differences between the studied infants. Estimation of FRC using helium dilution has also given lung volumes in healthy full term infants in the same range as those obtained by nitrogen dilution $(2,4)$. Furthermore, Boon et al. (4) found no difference in FRC determined by helium dilution at various breathing air oxygen concentrations.

In summary, the design of the method and the use of a computer bring about accurate and fast results, and enable further analysis of the obtained data. The method has also been found easy to use and is therefore suitable for clinical application.

\section{REFERENCES}

1. Beardsmore CS, Stocks J, Silverman M 1982 Problems in measurements of thoracic gas volume in infancy. J Appl Physiol Respirat Environ Exercise Physiol 52:995

2. Berglund G, Karlberg P 1956 Determination of the functional residual capacity in newborn infants. Act Paediatr 45:541

3. Bolton DPG, Cross KW, Kennaird DL 1970 Nitrogen washout estimations in newborn infants. J Physiol 208:6P

4. Boon AW, Ward-McQuaid JMC, Milner AD, Hopkin TE 1981 Thoracic gas volume, helium functional residual capacity and air-trapping in the first six hours of life: the effect of oxygen administration. Early Human Dev 5:157

5. DuBois AB, Botelho SY, Bedell GN, Marshall R, Comroe IH Jr A rapid plethysmographic method for measuring thoracic gas volume: a comparison with a nitrogen washout method for measuring function residual capacity in normal subjects. J Clin Invest 35:322 
6. Geubelle F, Francotte M, Beyer M, Louis I, Logvinoff MM 1977 Functional residual capacity and thoracic gas volume in normoxic and hyperoxic newborn infants. Act Paediatr Belg 30:221

7. Groom AC, Morin R, Fahri LE 1967 Determination of dissolved nitrogen in blood and investigation of nitrogen washout from the body. J Appl Physiol 23:706

8. Hanson JS, Shinozaki T 1970 Hybrid computer studies of ventilatory distribution and lung volume. Pediatrics 46:900

9. Henderson-Smart DJ, Read DJC 1979 Reduced lung volume during behavioral active sleep in the newborn. J Appl Physiol Respirat Environ Exercise Physiol 46:1081

10. Hjalmarson O 1974 Mechanics of breathing in newborn infants with pulmonary disease. Acta Paediatr Scand Suppl 247:10

11. Hlastala MP, Wranne B, Lenfant CJ 1973 Cyclical variations in FRC and other respiratory variables in resting man. J Appl Physiol 34:670

12. Lundin G 1953 Nitrogen elimination during oxygen breathing. Acta Physiol Scand Suppl 3:130

13. Nelson NM, Prod'hom LS, Cherry RB, Lipsitz PJ, Smith CA 1963 Pulmonary function in the newborn infant. V. Trapped gas in the normal infant's lung. $\mathrm{J}$ Clin Invest 42:1850

14. Prod'hom S, Levison H, Cherry RB, Drorbaugh JE, Hubbell JP, Smith CA
1964 Adjustment of ventilation, intrapulmonary gas exchange, and acidbase balance during the first day of life. Normal values in well-infants of diabetic mothers. Pediatrics 33:682

15. Richardson P, Galway W, Olson S, Bunell JB 1981 Computerized estimates of functional residual capacity in infants. Ann Biomed Eng 9:243

16. Robertson JS, Siri WE, Jones HB 1950 Lung ventilation patterns determined by analysis of nitrogen elimination rates: use of the mass spectrometer as a continuous gas analyzer. J Clin Invest 29:577

17. Saniie J, Saidel GM, Chester EH 1979 Real time moment analysis of pulmonary nitrogen washout. J Appl Physiol Respirat Environ Exercise Physiol 46:1184

18. Shinozaki T, Abajian J, Tabakin BS, Hanson JS 1965 Nitrogen washout computer. Am J Med Electron 4:23

19. Sjöqvist BA, Sandberg K, Hjalmarson O, Olsson T 1983 Assessment of lung volumes in newborn infants by a computer-assisted plethysmographic nitrogen washout method. Technical Report 4:83. Research Laboratory of Medical Electronics (Chalmers University of Technology, Göteborg, Sweden)

20. Strang LB, McGrath MW 1962 Alveolar ventilation in normal newborn infants studied by air wash-in after oxygen breathing. Clin Sci 23:129

21. Webster JG 1978 Medical Instrumentation: Application and Design. Houghton Mifflin Company, Boston

\title{
Ontogeny of Protection of Neonatal Mice from Lethal Herpes Simplex Virus Infection by Human Leukocytes, Antiviral Antibody, and Recombinant $\alpha$-Interferon
}

\author{
STEVE KOHL, ROBERT H. BIGELOW, AND LIAN S. LOO \\ Department of Pediatrics and Program in Infectious Diseases, University of Texas Medical School at Houston, \\ Houston, Texas 77025
}

\begin{abstract}
We have studied the peripheral blood leukocytes from human infants in an assay involving the protection of neonatal mice from herpes simplex virus (HSV) infection by human antibody, interferon, and leukocytes. Recombinant DNA $\alpha$-interferon (IFLrA), antibody, and Ficoll-Hypaque-purified mononuclear cells (MC) from human adults administered intraperitoneally protected neonatal mice from a lethal HSV challenge 1 day later (73.6\% survival). MC obtained from human infants less than 130 days old in combination with IFLrA and antibody afforded no protection (15.2\% survival; $p<$ 0.0005 compared to survival with adults' $M C)$. MC from infants over 130 days protected the neonatal mice $[60 \%$ survival; not significantly different from survival using adult cells, but significantly $(p<0.0005)$ different than survival using MC from younger infants]. The ontogeny of MC protection parallels the clinical development of resistance of infants to HSV infection. (Pediatr Res 18:1164$1167,1984)$
\end{abstract}

Received February 2, 1984; accepted April 19, 1984

Address correspondence and reprint requests to Dr. Steve Kohl, Program in Infectious Diseases, University of Texas Medical School, P.O. Box 20708, Houston TX 77025 .

This work was supported by National Institutes of Health Grant HD 13021 and a grant from the National Foundation, March of Dimes.

\author{
Abbreviations \\ HSV, herpes simplex virus \\ IFLrA, recombinant DNA human $\alpha$-interferon \\ MC, human mononuclear leukocytes \\ PFU, plaque-forming units \\ IP, intraperitoneal
}

Neonatal humans are unusually susceptible to severe and often fatal HSV infections (1). The immunologic defect that explains their susceptibility to this common virus in the first months of life with development of resistance in the second half of the first year of life remains to be elucidated $(1,2)$. As in humans, neonatal mice are extremely susceptible to fatal HSV infection (3). We have developed a murine model of neonatal HSV infection in which infant mice can be nearly completely protected from an otherwise lethal HSV infection by a combination of human antiviral antibody, human natural or recombinant DNA $\alpha$-interferon, and human mononuclear leukocytes (4-6). None of these factors by itself was protective. While leukocytes from adult humans could confer this protection, those obtained from umbilical cord blood were unable to protect in combination with either antibody (7) or antibody and interferon (4). We have now 